Check for updates

Cite this: DOI: 10.1039/c9re00309f

\title{
A mathematical model of a slurry reactor for the direct synthesis of hydrogen peroxide $\uparrow$
}

\author{
Fabio Pizzetti, Vittoria M. A. Granata, Umberto Riva, \\ Filippo Rossi iD* and Maurizio Masi*
}

\begin{abstract}
The direct synthesis of hydrogen peroxide represents a green alternative to the conventional large-scale anthraquinone process and offers a significantly economic advantageous way of producing a compound for which the global demand is ever increasing due to its multiple uses. However, the implementation of this process still faces important challenges regarding productivity, selectivity, and safety of this theoretically simple but practically not trivial reaction. In principle, we can smartly implement a process if we deeply know how the system and the reaction proceed. In this perspective, the importance of modeling the process itself becomes clear, and that is the purpose of this study: to develop a mathematical model for the direct synthesis of hydrogen peroxide in a continuous catalytic three-phase reactor. In particular, the fluid dynamic aspects of the system were studied, along with the kinetics and interphase mass exchange. Under our conditions, gas/liquid mass transfer prevailed, thus the reactor worked in a convective mass-transfer regime. Model equations were written and implemented in order to carry out different simulations and to obtain a first dimensioning of the reactor. It should be underlined that the definition of such a model can constitute a step forward and an opening of the doors for the industrial world to implement the direct synthesis of $\mathrm{H}_{2} \mathrm{O}_{2}$, ultimately helping make this process an effective industrial production.
\end{abstract}

Received 31st July 2019,

Accepted 11th October 2019

DOI: 10.1039/c9re00309f

rsc.li/reaction-engineering

\section{Introduction}

Hydrogen peroxide is a key compound in the sustainable chemical industry. This reactant has many applications in the textile, pulp/paper, wastewater treatment, and chemical industries, or even in soil remediation, disinfection, and sterilization processes. It is a powerful, attractive, and versatile oxidizing agent due to its "green" nature. Indeed, it decomposes to water and oxygen only, which gives it an exceptional pollution control ability and significantly decreases the end-user waste disposal cost. ${ }^{1}$

Actually, hydrogen peroxide is produced via the anthraquinone auto-oxidation (AO) process, where it is formed via a two-step process through an organic carrier molecule. ${ }^{2}$ Although this conventional process is a well-proven and reliable operating technology and has been the dominant method for $\mathrm{H}_{2} \mathrm{O}_{2}$ production since the middle of the twentieth century, it has several important drawbacks. ${ }^{3}$ The hydrogen peroxide must be produced in large amounts and at

Department of Chemistry, Materials and Chemical Engineering "Giulio Natta", Politecnico di Milano, via Mancinelli 7, 20131 Milan, Italy.

E-mail: filippo.rossi@polimi.it, maurizio.masi@polimi.it; Fax: (+39) 02 23993180, Fax: (+39) 02 23993180; Tel: (+39) 02 23993145, (+39) 0223993131

$\dagger$ Electronic supplementary information (ESI) available: Estimation of bubble diameter, energy balance, physico-chemical properties and model parameters. See DOI: $10.1039 / \mathrm{c} 9 \mathrm{re} 00309 \mathrm{f}$ concentrations of about $50 \mathrm{wt} \%$ or $70 \mathrm{wt} \%$ in order to afford economic feasibility, which requires an energy-intensive separation and concentration of $\mathrm{H}_{2} \mathrm{O}_{2}$. The high concentration is necessary to minimize the liquid volume, thus decreasing the transport expenses. End users of hydrogen peroxide though typically require a concentration in the range of $1-10 \mathrm{wt} \%$ and so this dilution represents an additional cost. ${ }^{4}$ Furthermore, this process requires big industrial plants, wastewater treatment, and produces a large amount of by-products. For these reasons, the cost of hydrogen peroxide is relatively high, limiting its industrial large-scale use. The global demand for hydrogen peroxide has been growing for the last decades, making it necessary to find several alternative ways of producing this chemical, e.g., via electrochemical synthesis. ${ }^{5-8}$ Despite this possibility, a direct synthesis (DS) of hydrogen peroxide in the presence of a catalyst and a solvent seems to be the best option, merely because in theory it is exceptionally easy, i.e., by just combining one molecule of hydrogen with one of oxygen and having water as the only by-product. This ecofriendly process reduces both operating and investment costs, providing an atomic-efficient, possibly small-scale on-site way of producing $\mathrm{H}_{2} \mathrm{O}_{2}$, avoiding the need to transport it in a concentrated form. ${ }^{9,10}$

Due to its potential, the DS process is a hot topic in the scientific community. It has been a long-time dream of chemists and chemical engineers and, to date, some big 
steps forward have been made. However, the real breakthrough has not happened yet, along with the commercialization of the process. That is, because the direct synthesis faces important technical challenges. First, hydrogen and oxygen form an explosive gas mixture over a wide range of concentrations $\left(5-96 \% \mathrm{H}_{2}\right.$ in $\left.\mathrm{O}_{2}\right) .{ }^{11}$ This has to be avoided by dilution of the gases, which leads to low concentrations in the liquid phase, reducing the overall rate of $\mathrm{H}_{2} \mathrm{O}_{2}$ production. Therefore, high pressures in the order of several thousand psi are required to increase the amount of hydrogen dissolved in water, which significantly increases the cost of the equipment and the operating costs. At these low concentrations, mass transfer of the gaseous reactants in the liquid phase plays a huge role and there are different studies that have focused particularly on this aspect. ${ }^{12-14}$ Besides the safety problem, another issue with the reaction is the presence of consecutive/parallel reactions, which influences the selectivity of the process. Numerous studies concerning the kinetics of these undesired reactions have been carried out. ${ }^{15,16}$ Biasi et $a .^{17}$ studied the decomposition, hydrogenation, and direct synthesis of hydrogen peroxide performed on a commercial $\mathrm{Pd} / \mathrm{C}$ catalyst, on which all three reactions take place, analyzing the temperature effects and subsequent hydrogen addition, without using promoters.

To overcome these problems, the development of new catalyst concepts seems to be very significant and a lot of work has been done in this sense. ${ }^{9,10,18}$ Even the addition of halides and acids as promoters and stabilizers was discovered to be useful for the reduction of the effects of undesired reactions, although this then leads to a serious complication in comparing different works. ${ }^{10,14,17,19}$ Another interesting strategy is that of focusing on the reactor technology. For instance, Voloshin et $a .^{11}$ safely produced hydrogen peroxide by the direct combination of hydrogen and oxygen in a microreactor in an explosive regime, achieving concentrations as high as $1.3 \mathrm{wt} \% \mathrm{H}_{2} \mathrm{O}_{2}$, and this was possible because much higher temperatures and pressures are required to start an explosion in a microchannel than in a macrochannel.

Selinsek et al. ${ }^{20}$ developed a novel intensified suspensionflow membrane microreactor system for the direct synthesis of hydrogen peroxide, and other works also have developed this kind of technology. ${ }^{1,4,12}$ The aforementioned idea seems useful and inspiring, but all in all, it becomes less enriching if a model including the kinetics and mass-transfer limitations is not considered. As the previous mentioned studies modeled the direct synthesis process, so our work would like to make a remarkable imprint in this overview. Our aim was the formulation of a mathematical model for the direct synthesis of hydrogen peroxide in a continuous catalytic three-phase reactor. The process was realized with a once-through flow of slurry made up of the solvent, namely water, and the catalyst, e.g., a supported catalyst, in which the active element consisted of Pt (or Pd). Hydrogen and oxygen were alternatively fed in to the reactor, so that when the second gaseous reagent (oxygen) was supplied, the first (hydrogen) was almost absent in the gas phase, being now adsorbed onto the catalyst. This expedient allowed operating outside the flammability limits, thereby ensuring safer conditions and promoting the desired reaction. For the same reason, the gaseous hold up had to be limited (a few percent), and the formation of large-sized bubbles had to be avoided. Then, even in the unfortunate scenario in which there might be the onset of an explosive reaction, its effects would be confined if the bubbles were small and completely segregated in the liquid phase, which is favored by a high pressure of hundreds of atmospheres. For this purpose, high velocities of the liquid phase become necessary and a cocurrent flow of slurry and gas is preferred. ${ }^{21}$ Modeling requires the description of some main physico-chemical phenomena. First, the dissolution of hydrogen with the achievement of thermodynamic equilibrium between the gas, liquid, and adsorbed phases. Then, the rate of the synthesis controlled by the transfer of oxygen. Finally, the bubble diameter evolution controlled by their coalescence and breakup, due to the turbulence.

In the present study, we analyzed the fluid dynamic aspects, such as bubble dynamics, bubble motion stability, and slurry suspensibility. We determined that the bubble motion was stable for high liquid to gas volumetric flow ratios (therefore, high liquid flows and a low hold-up of the gaseous phase). Then, we modeled the mass transfer, underlining how under our conditions, the gas/liquid mass transfer was the determining step. Always under the model conditions, we found the reactor to operate in a convective mass-transfer regime. For the liquid/solid mass transfer, external diffusion was shown to be decisive due to the small dimensions of the catalytic particles. Besides, we wrote equations for the system (also in dimensionless terms) and we implemented these equations, along with fluid dynamics, mass transfer, and kinetics mathematical expressions obtained. The mass-transfer properties that will be discussed in the following sections are different from the ones analyzed in other works, being that they are strictly related to the fluid properties and reactor setups employed herein. As already mentioned, microreactors were used, for instance, in the work of Voloshin et al. ${ }^{11}$ Thanks to the reduced dimension, the mass transfer here happened to be quicker with respect to conventional reactors, but there were problems related to catalyst dispersion and high pressure drops (due to the small dimensions of the catalytic particles). Selinsek et al. ${ }^{20}$ tried to overcome such a problem by coupling a single-phase microreactor with a membrane one, thus overcoming the problems related to the catalyst dispersion and keeping the advantage of a quick mass transfer. The slurry reactor studied here will instead work in the mass-transfer regime, since this is the rate-determining step, differently from the microreactor configuration. Being a three-phase reactor, different types of mass transfer were present, thus an overall mass-transfer coefficient was defined. Finally, some examples of simulations and a first dimensioning of the reactor are reported. 


\section{Model description}

In this section, the different aspects taken into account for the mathematical model are considered. Further details on the estimation of the bubbles' diameters, energy balance, physico-chemical properties, and model parameters are present in the ESI.†

\subsection{Fluid dynamics}

Being that the axial dimension of the reactor was bigger than its diameter $\left(d_{\mathrm{r}} / L \ll 1\right)$, the problem was assumed to be monodimensional. To ensure good contact between the bubbles and slurry, both phases were fed with an equal flow. A biphasic motion was obtained operating at high speed $(>2$ $\mathrm{m} \mathrm{s}^{-1}$ ) and having small gaseous hold-ups $(\varepsilon<0.1)$, leading to the formation of gas bubbles under $2 \mathrm{~mm}$, which could be decreased in size using a porous sparger (only with short tubes). This motion presents an elevated turbulence that keeps the size of the gas bubbles relatively small. The contribution of the gas phase relative velocity with respect to the liquid phase can be neglected. Using the forces conservation equation, it is possible to obtain the gas bubble terminal velocity: ${ }^{22}$

$$
\begin{gathered}
u_{\mathrm{t}}=\frac{g d_{\mathrm{b}}{ }^{2}\left(\hat{\rho}_{\mathrm{L}}-\hat{\rho}_{\mathrm{G}}\right)}{36 \mu_{\mathrm{L}}} \quad \operatorname{Re}<75 \\
u_{\mathrm{t}}=1.75 \sqrt{\frac{g d_{\mathrm{b}}\left(\hat{\rho}_{\mathrm{L}}-\hat{\rho}_{\mathrm{G}}\right)}{\hat{\rho}_{\mathrm{L}}}} \quad \operatorname{Re}>75
\end{gathered}
$$

where $g, d_{\mathrm{b}}, \mu_{\mathrm{L}}, \hat{\rho}_{\mathrm{L}}$, and $\hat{\rho}_{\mathrm{G}}$ are the acceleration of gravity, the diameter of the bubbles, the viscosity, and the density of the liquid phase, and the density of the gaseous phase respectively. Eqn (1a) and (1b) are valid only if the bubble dimension is small; if it increases over a diameter of $5 \mathrm{~mm}$, an asymptotic value of approximately $25 \mathrm{~cm} \mathrm{~s}^{-1}$ is reached.

The upward traveling-velocity has a vector component with the liquid interstitial velocity $v_{\mathrm{L}}$ and they combine to form the interstitial velocity in the gaseous phase $v_{\mathrm{G}}:^{23}$

$$
v_{\mathrm{G}}=v_{\mathrm{L}}+u_{\mathrm{t}} \cos \beta
$$

where $\beta$ is the angle between the vertical line and the liquid phase's velocity direction: if $\beta=0$, the motion is equal and is an upflow; if $\beta=\pi$, the motion is equal and is a downflow, and if $\beta=\pi / 2$ the motion is equal and horizontal. The gaseous phase hold-up is calculated using the following equation:

$$
\varepsilon=\frac{u_{\mathrm{G}}}{v_{\mathrm{L}}+u_{\mathrm{t}} \cos \beta}
$$

The interstitial velocity of the liquid phase can be calculated computing the ratio between the superficial velocity and its hold-up:

$$
v_{\mathrm{L}}=\frac{u_{\mathrm{L}}}{1-\varepsilon}
$$

Substituting eqn (3) in (4), a second-order algebraic equation is obtained, whose solutions are the hold-up values of the gaseous phase as a function of the two superficial velocities and the angle $\beta$ :

$$
u_{\mathrm{t}} \cos \beta \cdot \varepsilon^{2}-\left(u_{\mathrm{t}} \cos \beta+u_{\mathrm{L}}+u_{\mathrm{G}}\right) \varepsilon+u_{\mathrm{G}}=0
$$

In the hypothesis of horizontal tubes, the hold-up is directly obtained from the ratio between the volumetric flow rates of the two separate phases:

$$
\frac{u_{\mathrm{G}}}{u_{\mathrm{L}}}=\frac{\varepsilon}{1-\varepsilon}
$$

The most favorable fluid dynamic conditions are vertical descending flows. Unfortunately, due to the excessive reactor length, descending and ascending segments should be alternated. Thus, in the following dissertation, the configuration analyzed was composed only of horizontal tubes, where interstitial velocity gradients between the phases were not present.

Therefore, as can be deduced from eqn (5), the superficial velocities for single phases can be determined from the slurry velocity:

$$
\begin{gathered}
u_{\mathrm{sl}}=\frac{Q_{\mathrm{sl}}}{\Omega} \\
u_{\mathrm{L}}=\frac{Q_{\mathrm{L}}}{\Omega}=(1-\alpha) u_{\mathrm{sl}} \\
u_{\mathrm{G}}=\frac{Q_{\mathrm{G}}}{\Omega}=\frac{\varepsilon}{1-\varepsilon} u_{\mathrm{sl}} \\
u_{\mathrm{p}}=\frac{Q_{\mathrm{p}}}{\Omega}=\alpha u_{\mathrm{sl}}
\end{gathered}
$$

where $Q, u_{\mathrm{sl}}, \Omega, \alpha$, and $\varepsilon$ are the volumetric flow rates, the slurry superficial velocity, the reactor cross section, and the volumetric hold-ups of the catalyst in the slurry and the gas, respectively. The gas hold-up can be calculated using the following formula:

$$
\varepsilon=\frac{Q_{\mathrm{G}}}{Q_{\mathrm{G}}+Q_{\mathrm{sl}}}
$$

Eqn (11) is compatible with the condition $\varepsilon \rightarrow 0$ if $Q_{\mathrm{G}} \rightarrow 0$. The presence of a solid in suspension does not alter the hold-up value if the solid accounts for less or equal to $10 \%$ of the overall weight. For higher values, the hold-up decreases when increasing the weight of the solid in suspension. This also affects the values of the gas/liquid interfacial area, which decreases with a solid weight above $10 \%$ overall. Under this value, all the parameters are not affected. The discussion above can be even more validated achieving a modest correction by replacing the properties of the slurry with those of the liquid phase. ${ }^{24}$ 


\subsection{Stability of bubble motion}

State diagrams provide a good first analysis to define the stability region of the biphasic motion of the reaction bubbles. Horizontal tubes have been shown to follow Barker's diagram approximation, ${ }^{25}$ whose stability conditions are calculated with the following equations:

$$
\begin{gathered}
\frac{\hat{\rho}_{\mathrm{L}} Q_{\mathrm{L}}}{\hat{\rho}_{\mathrm{G}} Q_{\mathrm{G}}}>200 \\
\frac{\hat{\rho}_{\mathrm{G}} Q_{\mathrm{G}}}{\lambda}>12.289\left(\frac{\hat{\rho}_{\mathrm{G}} Q_{\mathrm{G}}}{\lambda \psi \hat{\rho}_{\mathrm{L}} Q_{\mathrm{L}}}\right)
\end{gathered}
$$

where $\lambda=\sqrt{\hat{\rho}_{\mathrm{G}}^{\prime} \hat{\rho}_{\mathrm{L}}^{\prime}} \approx \sqrt{P}$ and $\psi=\left(\sqrt[3]{\mu_{\mathrm{L}}^{\prime} / \rho_{\mathrm{L}}^{\prime 2}}\right) / \sigma^{\prime} \approx 1$ are two dimensionless parameters based on reduced physical properties given by the ratio between the properties of the reacting fluids and the properties of the air/water system at atmospheric pressure.

In this analysis, the first parameter coincided with the root of the operative pressure, and the second was basically unitary. Bubble motion was stable for high flow values between the liquid and gas (high liquid flow rates and low hold-ups).

\subsection{Bubble diameter evaluation}

The size of the bubbles is a function of the flow dynamics inside the reactor and the properties of the sparger that produces dispersion in the gas phase. There are a few correlations that allow an appraisal of the bubble diameter, where Sauter's estimation is relevant in the scenario under consideration, as it allows direct evaluation of the interphasic area. $^{25}$ In a very turbulent system, such as the one here described, the bubble size $d_{\mathrm{b}}$ can be determined using the turbulent dissipation of the energy $e_{\mathrm{D}}$, which limits the maximum stable bubble dimension: ${ }^{26-29}$

$$
d_{\mathrm{b}}=\frac{2}{e_{\mathrm{D}}^{0.4}}\left(\frac{\sigma}{\rho \hat{\mathrm{L}}}\right)^{0.6} \varepsilon^{0.5}\left(\frac{\mu_{\mathrm{G}}}{\mu_{\mathrm{L}}}\right)
$$

where $\sigma$ is the interfacial tension, $\mu_{\mathrm{G}}$ is the gas phase viscosity, and $\mu_{\mathrm{L}}$ is the liquid phase one. The turbulent dissipation intensity can be estimated from Kolmogorov's theory as a function of the liquid phase superficial velocity $\mu_{\mathrm{L}}$ and the diameter of the tube $d_{\mathrm{R}}$ :

$$
e_{\mathrm{D}} \cong u_{\mathrm{L}}^{3} / d_{\mathrm{R}}
$$

Sauter's diameter is a useful engineering representation, valid on first approximation. In general, it can be observed that when increasing the gaseous flow, the bubble dimension increases too. Instead, an opposite effect occurs when the operating pressure is increased, while the temperature effect is negligible. ${ }^{28}$

\subsection{Interphasic area estimation}

The gas/liquid interfacial area can be computed if knowing the Sauter's bubble diameter: ${ }^{24}$

$$
a_{\mathrm{b}}=\frac{6 \varepsilon}{d_{\mathrm{b}}}
$$

As far as the solid/liquid interphasic area is concerned, it can be evaluated using the following equations:

$$
\begin{array}{r}
a_{\mathrm{p}}=\frac{6}{d_{\mathrm{p}}} \\
a_{\mathrm{c}}=\alpha(1-\varepsilon) a_{\mathrm{p}}
\end{array}
$$

where $d_{\mathrm{p}}$ and $\alpha$ represent the average diameter of a catalytic particle and the volumetric catalytic fraction in the slurry. The $\alpha$ parameter is calculated from the catalytic mass fraction knowing the densities of the dry catalyst $\hat{\rho}_{\mathrm{S}}\left(1-\varepsilon_{\mathrm{p}}\right)$ and the slurry $\hat{\rho}_{\mathrm{sl}}$ :

$$
\alpha=W \frac{\hat{\rho}_{\mathrm{sl}}}{\hat{\rho}_{\mathrm{S}}\left(1-\varepsilon_{\mathrm{p}}\right)}
$$

The slurry density is calculated knowing the density of the liquid and the solid catalyst:

$$
\hat{\rho}_{\mathrm{sl}}=\left[\frac{1-W}{\hat{\rho}_{\mathrm{L}}}+\frac{W}{\hat{\rho}_{\mathrm{S}}}\right]^{-1}
$$

Analyzing eqn (20a), we can see that the density of the slurry is independent of the porosity of the particle. The proof of this equation can be achieved accounting for the fact that, during the formation of the slurry in the reactor, the liquid phase penetrates also in to the pores of the particles, and the final volume of the slurry is determined from the volume of the particles and the liquid on the outside. The volumes per unit mass of the totality of the liquid phase, the liquid contained in the pores, and the catalytic particle are, respectively, $(1-W) / \hat{\rho}_{\mathrm{L}}, \varepsilon_{\mathrm{p}} W / \hat{\rho}_{\mathrm{S}}\left(1-\varepsilon_{\mathrm{p}}\right)$ and $W / \hat{\rho}_{\mathrm{S}}\left(1-\varepsilon_{\mathrm{p}}\right)$, which can be arranged as in eqn (20b) to give eqn (20a).

$$
\hat{\rho}_{\mathrm{sl}}=\left[\frac{1-W}{\hat{\rho}_{\mathrm{L}}}-\frac{\varepsilon_{\mathrm{p}} W}{\hat{\rho}_{\mathrm{S}}\left(1-\varepsilon_{\mathrm{p}}\right)}+\frac{W}{\hat{\rho}_{\mathrm{S}}\left(1-\varepsilon_{\mathrm{p}}\right)}\right]
$$

The first two terms in square brackets in eqn (20b) are the external liquid contribution.

\subsection{Suspension properties of the slurry}

Since there is a solid suspended in the liquid phase, we need to check the flow dynamics that allow its suspension and the maximum weight of solid that can be added to the slurry. This information has an important role in the process. A useful equation for calculating its value is the following: ${ }^{24}$

$$
\frac{u_{\mathrm{G}}^{\min }}{u^{*}}=0.801\left(\frac{\hat{\rho}_{\mathrm{p}}-\hat{\rho}_{\mathrm{L}}}{\hat{\rho}_{\mathrm{L}}}\right)^{0.6}\left(\frac{W \hat{\rho}_{\mathrm{sl}}}{\hat{\rho}_{\mathrm{p}}}\right)^{0.146}\left(\frac{\sqrt{g d_{\mathrm{R}}}}{u^{*}}\right)^{0.24}\left[1+807\left(\frac{g \mu_{\mathrm{sl}}^{4}}{\hat{\rho}_{\mathrm{sl}} \sigma^{3}}\right)\right]^{0.578}
$$


where $u^{*}$ is the maximum velocity of the decantation of solid particles (determined through an equation similar to eqn (1a)) and $W$ is the massive fraction of catalyst in the slurry.

\section{Interphase mass transfer}

Reactants are injected in to the reactor in the gaseous phase, while the catalyst is finely spread in the aqueous phase; thus the model should account for every type of interphasic mass transfer. $^{24,30}$ In the following, the values of molar fractions near the interface will be denoted with an asterisk, thus the gas phase driving force is expressed by $y_{i}-y_{i}^{*}$, the liquid ones by $x_{i}^{*}-x_{i}$ and $x_{i}-x_{i}^{* \mathrm{p}}$, and the intraparticle transfer by $\vartheta_{i}-\vartheta_{i}^{*}$. With $\vartheta_{i}$, the catalyst fractional occupancy at thermodynamic equilibrium is indicated.

\subsection{Gas/liquid mass transfer}

This case study presents small gas bubbles, and all the resistances are concentrated in the liquid phase. The interphasic flow $J_{i}$ is diffusive and is obtained using the following equation. ${ }^{24,26,30}$

$$
J_{i}=k_{\mathrm{L}, i} \hat{\rho}_{\mathrm{L}}\left(\frac{y_{i}}{K_{i}}-x_{i}\right)
$$

where $k_{\mathrm{L}, i}, \tilde{\rho}_{\mathrm{L}}, y_{i}, x_{i}$, and $K_{i}$ are the heat-transfer coefficient of the liquid phase, the molar density of the liquid phase, the molar fractions of the $i$-th species in the gaseous phase and liquid phase, and the phase-partitioning equilibrium constant, respectively. The expression for the evaluation of $K_{i}$ changes depending on the component conditions (if subcritic or super-critic): ${ }^{25}$

$$
\begin{gathered}
K_{i}=\frac{P_{i}^{\circ}(T)}{P} \quad\left(\text { sub-critic components: } \mathrm{H}_{2} \mathrm{O}, \mathrm{H}_{2} \mathrm{O}_{2}\right) \\
K_{i}=\frac{H_{i}(T)}{P} \quad\left(\text { super-critic components: } \mathrm{H}_{2} \mathrm{O}, \mathrm{O}_{2}, \mathrm{~N}_{2}\right)
\end{gathered}
$$

where $P, P_{i}^{\circ}(T)$, and $H_{i}(T)$ are the operating pressure, the vapor tension, and Henry's constant for the components analyzed.

Eqn (22) is valid under the assumption of an ideal mixture assuming the solutions to be simple and ideal, but in the case of a real mixture, activity coefficients should be inserted there. To determine the mass-transfer coefficient, several formulas can be used based on different hypotheses. For high-turbulence systems, ${ }^{24}$ the theory of turbulent dissipation can be used:

$$
k_{\mathrm{L}, i}=0.13 \mathrm{Sc}^{-0.667}\left[\frac{e_{\mathrm{D}} \mu_{\mathrm{L}} g}{\hat{\rho}_{\mathrm{L}}^{2}}\right]^{0.25}
$$

For small bubbles $\left(d_{\mathrm{b}}<2.5 \mathrm{~mm}\right)$, we can use the correlation from Chalderbank et al.: ${ }^{25}$

$$
\mathrm{Sh}=\frac{k_{\mathrm{L}, i} d_{\mathrm{b}}}{D_{i}}=2+0.31 \mathrm{Ra}^{1 / 3}
$$

The last scenario is when small differences between the relative velocities are observed; this situation can be studied applying the penetration theory: ${ }^{25}$

$$
k_{\mathrm{L}, i}=\frac{10 D_{i}}{d_{\mathrm{b}}}
$$

\subsection{Liquid-solid mass transfer}

Two different types of transports are present at a liquid-solid interface: diffusion in the external boundary layer and intraparticle diffusion. Under this hypothesis, a bimodal porosity distribution function is considered. At higher porosity (macropores), the internal diffusion is dominant, while at a low level of porosity (micropores), the adsorption process prevails. The adsorption property is defined with the catalyst loading capacity $\Gamma_{i}^{\infty}$. Given the small size of the catalytic particle, the simplified "SOL model" has been adopted here, where the amount of mass inside the particle corresponds to the adsorbed concentration $q_{i}\left(\mathrm{~mol} \mathrm{~cm}{ }^{-3}\right){ }^{31,32}$

$$
q_{i}=\left(1-\varepsilon_{\mathrm{p}}\right) \hat{\rho}_{\mathrm{S}} \Gamma_{i}^{\infty} \vartheta_{i}+\varepsilon_{\mathrm{p}} \widetilde{\rho}_{\mathrm{L}} x_{i}^{* \mathrm{p}}
$$

where $\varepsilon_{\mathrm{p}}$ is the catalyst porosity. Eqn (27) takes into account both the species adsorbed onto the micropores and those contained in the macropores. The small size of the catalytic particle makes it possible to neglect the effect of diffusion in the macropores and to assimilate the composition of the liquid phase to the surface composition $x_{i}^{* \mathrm{p}}$. This means that the catalyst has a unitary efficiency, which is in accordance with the efficiency of a reactor operating under a diffusive external regime. Given the low solubility of the gaseous compounds in the liquid phase, the amount of them contained inside the macropores of the particle is negligible compared to the amount adsorbed by the catalyst active phase. Under these conditions, the mass flow rate between the liquid and adsorbed phase is: ${ }^{33}$

$$
N_{i}=k_{\mathrm{eff}, i}\left(q_{i}-q_{i}^{*}\right)=k_{\mathrm{eff}, i} \hat{\rho}_{\mathrm{S}} \Gamma_{i}^{\infty}\left(1-\varepsilon_{\mathrm{p}}\right)\left(\vartheta_{i}-\vartheta_{i}^{*}\right)
$$

In eqn (28), the effective mass-transfer coefficient $k_{\text {eff }, i}$ accounts for both contributions previously discussed (liquid diffusion $k_{\mathrm{s}, i}$ and intraparticle diffusion $k_{\mathrm{p}, i}$ ):

$$
k_{\mathrm{eff}, i}=\left[\frac{1}{k_{\mathrm{p}, i}}+\frac{1}{k_{\mathrm{s}, i}}\right]^{-1}
$$

In this case study, given the small size of the catalytic particle, external diffusion was more critical.

We also needed to determine the equilibrium value of the fractional occupancy $\vartheta_{i}$. In this study, some information was not available so the Langmuir multi-component isothermal adsorbing curve was used:

$$
\vartheta_{i}^{*}=\frac{b_{i} x_{i}}{1+\sum_{j}} b_{j} x_{j}
$$

where $b_{i}$ indicates the equilibrium constant of adsorption $\left(b_{i}=b_{i}^{\circ} e^{\Delta H_{\text {ads }} / \mathrm{RT}}\right)$. We referenced the multicomponent 
equation to highlight the competition among all the chemical species relative to the adsorbing process. The amount of mass effectively adsorbed on the catalyst is sensitive to the fractional occupancy $\vartheta_{i}^{*}$, a thermodynamic parameter of an experimental nature. The trend of $\vartheta_{i}^{*}$ is shown in Fig. 1.

Both external and intraparticle exchange coefficients should be determined. The former can be computed using Glueckauf's equation: ${ }^{32}$

$$
k_{\mathrm{p}, i}=\frac{10 D_{\mathrm{eff}, i}}{d_{\mathrm{p}}}
$$

The effective diffusion coefficient of a particle is defined as:

$$
D_{\mathrm{eff}, i}=\frac{\varepsilon_{\mathrm{p}} D_{i}}{\tau}
$$

where $\tau$ is the tortuosity of the pores. For the external coefficient, we can use eqn (24) and (26) but with the particle diameter instead of the bubble one. To obtain the density of the dispersed phase, the density of the wet catalyst $\hat{\rho}_{\mathrm{p}}=\hat{\rho}_{\mathrm{S}}(1-$ $\left.\varepsilon_{\mathrm{p}}\right)+\varepsilon_{\mathrm{p}} \hat{\rho}_{\mathrm{L}}$ can be used.

\section{Kinetics}

The mechanism involves three catalytic reactions (the direct production of hydrogen peroxide, water formation, water oxidation) plus other decomposition reactions involving hydrogen peroxide in the liquid phase and the combustion of gaseous oxygen molecules. These last two reactions are negligible in this system and so they will not be included in the following dissertation; thus only catalytic reactions will be present using a Langmuir-Hinshelwood mechanism. The reaction rate is found using the equation:

$$
\widetilde{r}_{\mathrm{k}}^{\mathrm{S}}=k_{\mathrm{k}} \prod_{j=1}^{\mathrm{NCS}} \Gamma \circ j k=k_{\mathrm{k}} \prod_{j=1}^{\mathrm{NCS}}\left(\Gamma_{j}^{\infty} \vartheta_{j}\right)^{\circ j k}
$$

The reaction velocity constant can be calculated with the Arrhenius equation.

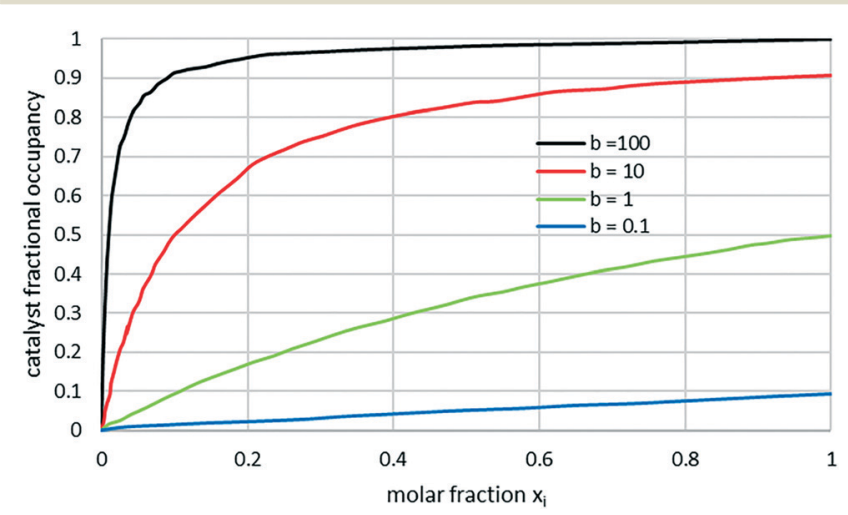

Fig. 1 Trend of the fractional occupancy $\vartheta_{i}^{*}$ as a function of the molar fraction of the liquid phase in contact with solid $x_{i}$ at different values of $b_{i}$.

\section{Reactor simulation}

The reactor in this case study basically consisted of two segments: in the first one, hydrogen was adsorbed onto the catalyst, while in the second one, oxygen was adsorbed. If there is a fixed amount of catalyst inside the suspension, then there will also be a fixed amount of fueling gas to the reactor. This also corresponds to the maximum amount adsorbed by the entire catalyst;

$$
F_{\mathrm{ads}, i}=\Omega u_{\mathrm{sl}} \alpha\left(1-\varepsilon_{\mathrm{p}}\right) \hat{\rho}_{\mathrm{S}} \Gamma_{i}^{\infty}=Q_{\mathrm{G}}^{\circ} \tilde{\rho}_{\mathrm{G}}^{\circ}
$$

If the desired productivity of hydrogen peroxide is known, then also the number of segments composing the reactor are known. This can be computed by the ratio between the moles of all the gaseous species adsorbed onto the catalyst and the maximum amount of adsorbed compounds for every reactor segment:

$$
\begin{gathered}
\dot{n}_{\mathrm{H}_{2} \mathrm{O}_{2}}=\frac{G_{\mathrm{H}_{2} \mathrm{O}_{2}}}{M_{\mathrm{H}_{2} \mathrm{O}_{2}}}=\dot{n}_{\mathrm{H}_{2}}=\dot{n}_{\mathrm{O}_{2}} \\
N=\frac{\dot{n}_{\mathrm{H}_{2}}+\dot{n}_{\mathrm{O}_{2}}}{\dot{F}_{\text {ads }}}
\end{gathered}
$$

The number of segments and the length of each of them allow us to determine the space necessary to complete the fueling gas adsorbing process. The length depends only on the mass-transfer coefficients, in particular the gas/liquid one. If we want to build a reactor to produce $4540 \mathrm{~kg} \mathrm{~h}^{-1}$ of $\mathrm{H}_{2} \mathrm{O}_{2}$ at $7 \%$ via a slurry of $5 \%$ in catalyst weight, the first segment can be sized as follow:

$$
\begin{gathered}
G_{\mathrm{H}_{2} \mathrm{O}_{2}}=4540 \mathrm{~kg} \mathrm{~h}^{-1}=1261 \mathrm{~g} \mathrm{~s}^{-1} \\
\dot{n}_{\mathrm{H}_{2} \mathrm{O}_{2}}=\frac{G_{\mathrm{H}_{2} \mathrm{O}_{2}}}{M_{\mathrm{H}_{2} \mathrm{O}_{2}}}=\dot{n}_{\mathrm{H}_{2}}=\dot{n}_{\mathrm{O}_{2}}=\frac{1261}{34}=37.092 \mathrm{~mol} \mathrm{~s}^{-1} \\
Q_{\mathrm{sl}}=\frac{G_{\mathrm{H}_{2} \mathrm{O}_{2}}}{w_{\mathrm{H}_{2} \mathrm{O}_{2}} \hat{\rho}_{\mathrm{sl}}} \approx \frac{4540}{0.07 \cdot 1000}=64.857 \mathrm{~m}^{3} \mathrm{~h}^{-1}=18.02 \mathrm{~L} \mathrm{~s}^{-1} \\
F_{\mathrm{ads}, i}=Q_{\mathrm{sl}} \alpha\left(1-\varepsilon_{\mathrm{p}}\right) \hat{\rho}_{\mathrm{S}} \Gamma_{i}^{\infty}=0.4635 \mathrm{~mol} \mathrm{~s}^{-1} \\
Q_{\mathrm{G}}^{\circ}=\frac{F_{\mathrm{ads}, i}}{\widetilde{\rho}_{\mathrm{G}}^{\circ}}=37.378 \mathrm{Nm}^{3} \mathrm{~h}^{-1} \\
N=\frac{2 \dot{n}_{\mathrm{H}_{2} \mathrm{O}_{2}}}{F_{\mathrm{ads}, i}} \approx 164 \text { segments }^{\circ}
\end{gathered}
$$

All the physical quantities reported here are proportional to the catalyst fraction in the slurry. If we double the amount of catalyst, the loading capacity of the fueling gas will double, and the number of segments will halve. The real inlet gas flow rate was evaluated starting from the ideal one knowing the process yield $\eta$ :

$$
Q_{\mathrm{G}, \mathrm{F}}=\frac{Q_{\mathrm{G}}^{\circ}}{\eta}
$$


Table 1 Baseline simulation boundary conditions

\begin{tabular}{|c|c|c|c|}
\hline Number of segments & 2 & $\begin{array}{l}\text { Catalyst particle } \\
\text { diameter }[\mu \mathrm{m}]\end{array}$ & 50 \\
\hline Length, $D_{\text {int }}, D_{\text {ext }}[\mathrm{m}]$ & $\begin{array}{l}100,0.10 \\
0.105\end{array}$ & $\begin{array}{l}\text { Coolant temperature } \\
{\left[{ }^{\circ} \mathrm{C}\right]}\end{array}$ & 0 \\
\hline Roughness [mm] & 2 & Pressure [atm] & 150 \\
\hline $\begin{array}{l}\text { Slurry volumetric flow } \\
\text { rate }\left[\mathrm{m}^{3} \mathrm{~h}^{-1}\right]\end{array}$ & 65 & $\begin{array}{l}\text { Gas volumetric flow } \\
\text { rate }\left[\mathrm{Nm}^{3} \mathrm{~h}^{-1}\right]\end{array}$ & 100 \\
\hline $\begin{array}{l}\mathrm{H}_{2} \mathrm{O} \text { inlet molar } \\
\text { fraction }\end{array}$ & 1.0 & $\begin{array}{l}\text { Inlet gas purity } \\
\left(\text { in } \mathrm{N}_{2} \text { ) }\right.\end{array}$ & $\begin{array}{l}99 \% \mathrm{H}_{2}, \\
98 \% \mathrm{O}_{2}\end{array}$ \\
\hline $\begin{array}{l}\text { Catalyst weight } \\
\text { fraction }\end{array}$ & $10 \%$ & $\begin{array}{l}\text { Gas bubble diameter } \\
{[\mu \mathrm{m}]}\end{array}$ & 1500 \\
\hline
\end{tabular}

The last part of reactor sizing involves the evaluation of its diameter and every segment length. The former depends only on the bubble motion stability.

As an example, some simulations were carried out employing the following conditions (Table 1).

\subsection{Variation in the bubble diameter}

The results obtained using the baseline simulation boundary conditions are shown in Fig. 2. It is possible to notice how $100 \mathrm{~m}$ was necessary to solubilize all the $\mathrm{H}_{2}$, whose composition stayed almost constant throughout the first segment, while the gaseous hold-up varied significantly along the same segment. Injecting $\mathrm{O}_{2}$ instantaneously altered the composition of the gaseous phase, which further changed due to $\mathrm{H}_{2}$ de-solubilization, thus regenerating the thermodynamic equilibrium. In the second section, hydrogen peroxide was formed and the liquid phase changed in composition. The temperature did not change significantly in the first segment, while it increased by approximately $3{ }^{\circ} \mathrm{C}$ due to heat release in the second part. The effect of the bubbles dimension was studied; practically their diameter can be decreased using a sparger. Simulations results obtained when decreasing the bubble diameter up to $500 \mu \mathrm{m}$ are shown in Fig. 3. Further simulations were carried out changing the diameter up to $100 \mu \mathrm{m}$, showing that the decrease in reactor length is directly proportional to the increase in the interfacial area.

\subsection{Pressure variation}

Another parameter that influences the length of solubilization is the pressure. When we changed the pressure, we detected an almost linear variation of the solubilization length, as can be observed in Fig. 4. The overall effect was almost similar to the one obtained changing the bubbles' dimension, thus reducing the solubilization length. The pressure had no impact on the temperature, having the same trend as in the baseline case. This allowed us to deduce that also the pressure could play a role in the dimensioning of the reactor.

\subsection{Increasing the number of stages}

Referring to the baseline conditions, a simulation with an 82-segments reactor was carried out to confirm the

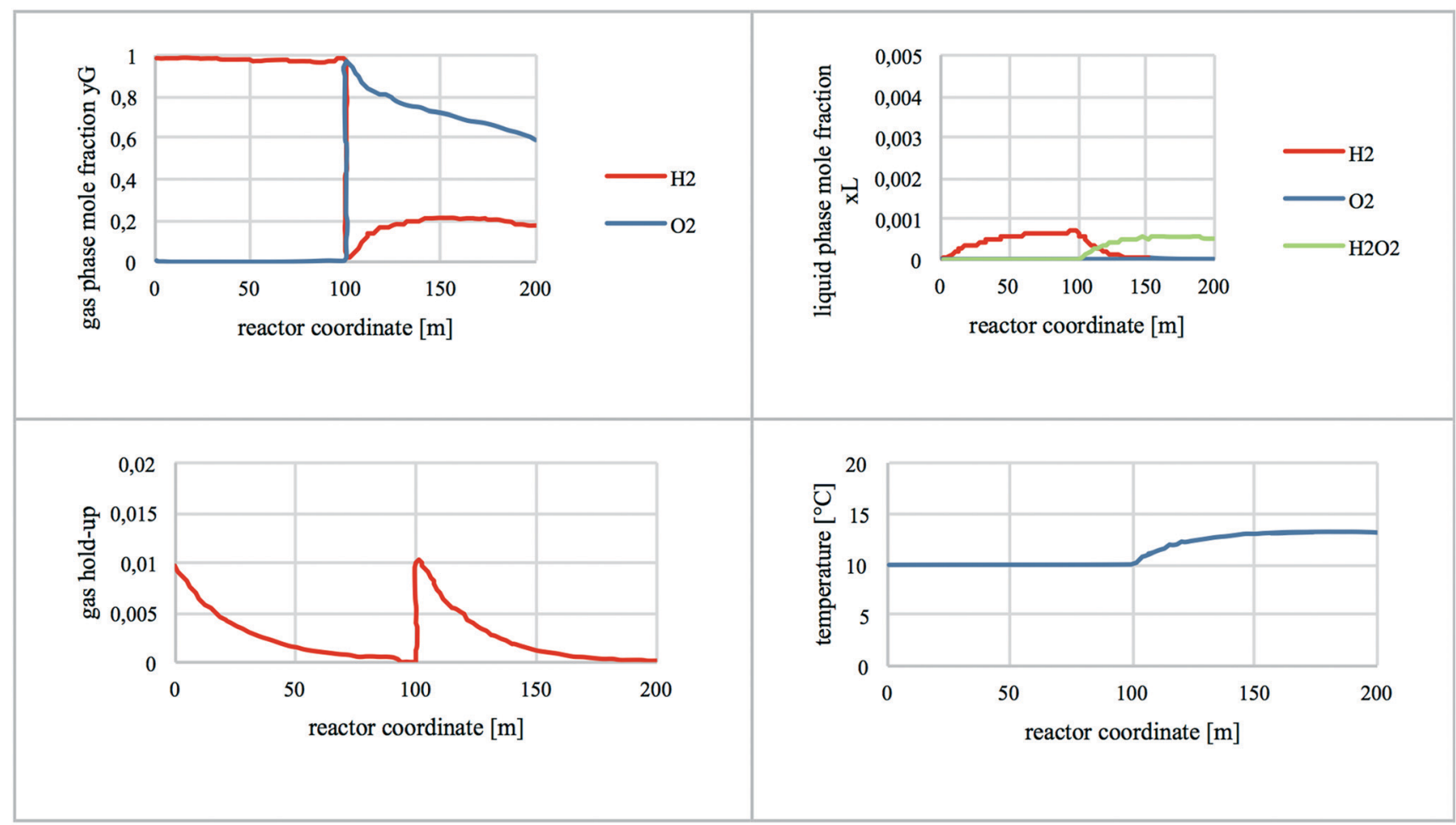

Fig. 2 Trend in the composition of the gaseous phase and liquid phase, of the hold-up, and of the slurry temperature along the axial reactor coordinate. Two-segment reactor, $d_{\mathrm{b}}=1500 \mu \mathrm{m}, P=150 \mathrm{~atm}$ (baseline). 


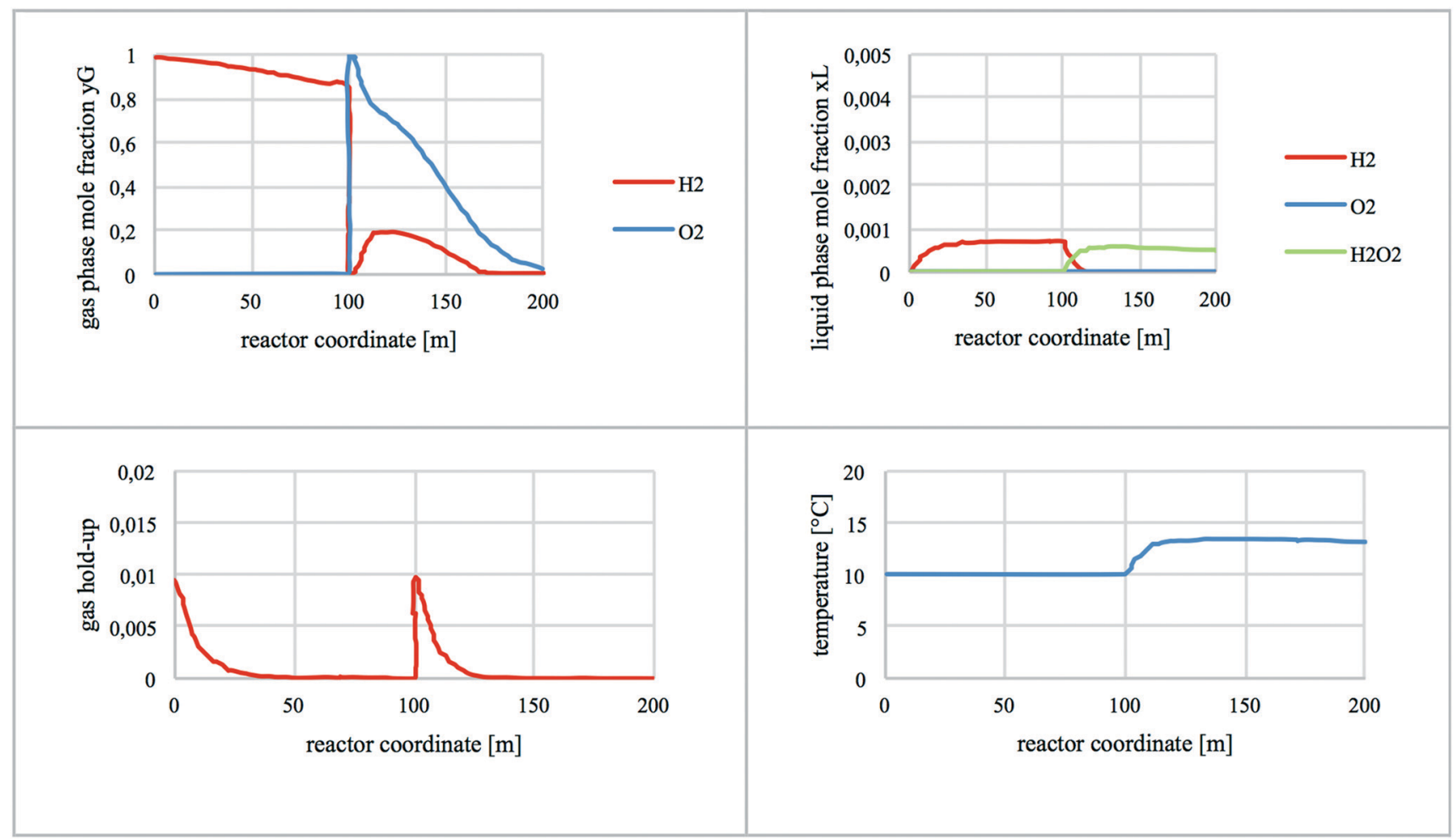

Fig. 3 Trend in the composition of the gaseous phase and liquid phase and the temperature of the slurry along the axial reactor coordinate. Two segment-reactor, $d_{\mathrm{b}}=500 \mu \mathrm{m}, P=150 \mathrm{~atm}$.

quantitative generation of hydrogen peroxide. The results are summed up in Fig. $5-8$. It is possible to notice the cyclic behavior of this setup every two reactor segments, within which the trends were the same obtained with the

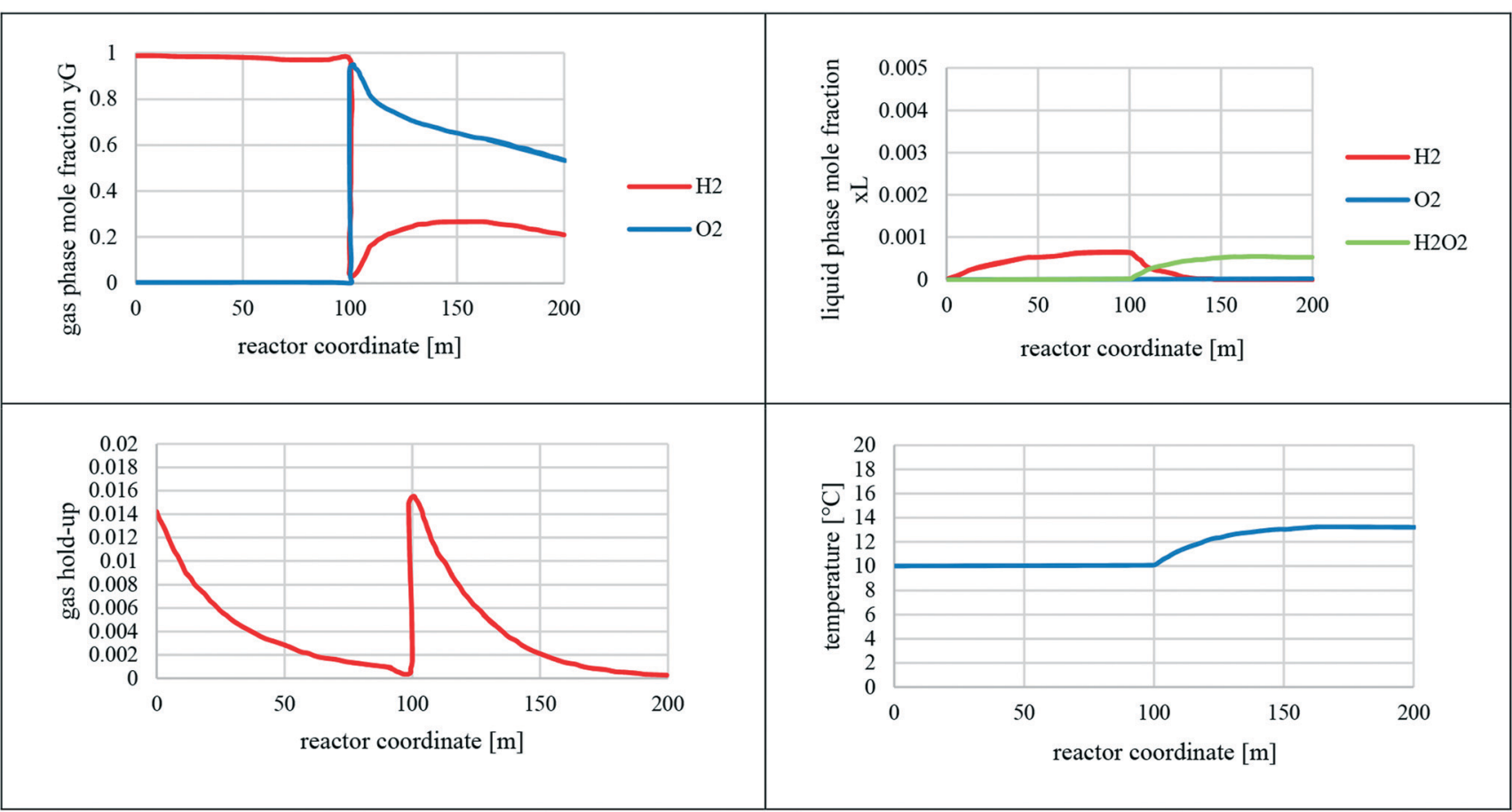

Fig. 4 Trend in the composition of the gaseous phase and liquid phase and the temperature of the slurry along the axial reactor coordinate. Two segment-reactor, $d_{\mathrm{b}}=1500 \mu \mathrm{m}, P=100 \mathrm{~atm}$. 


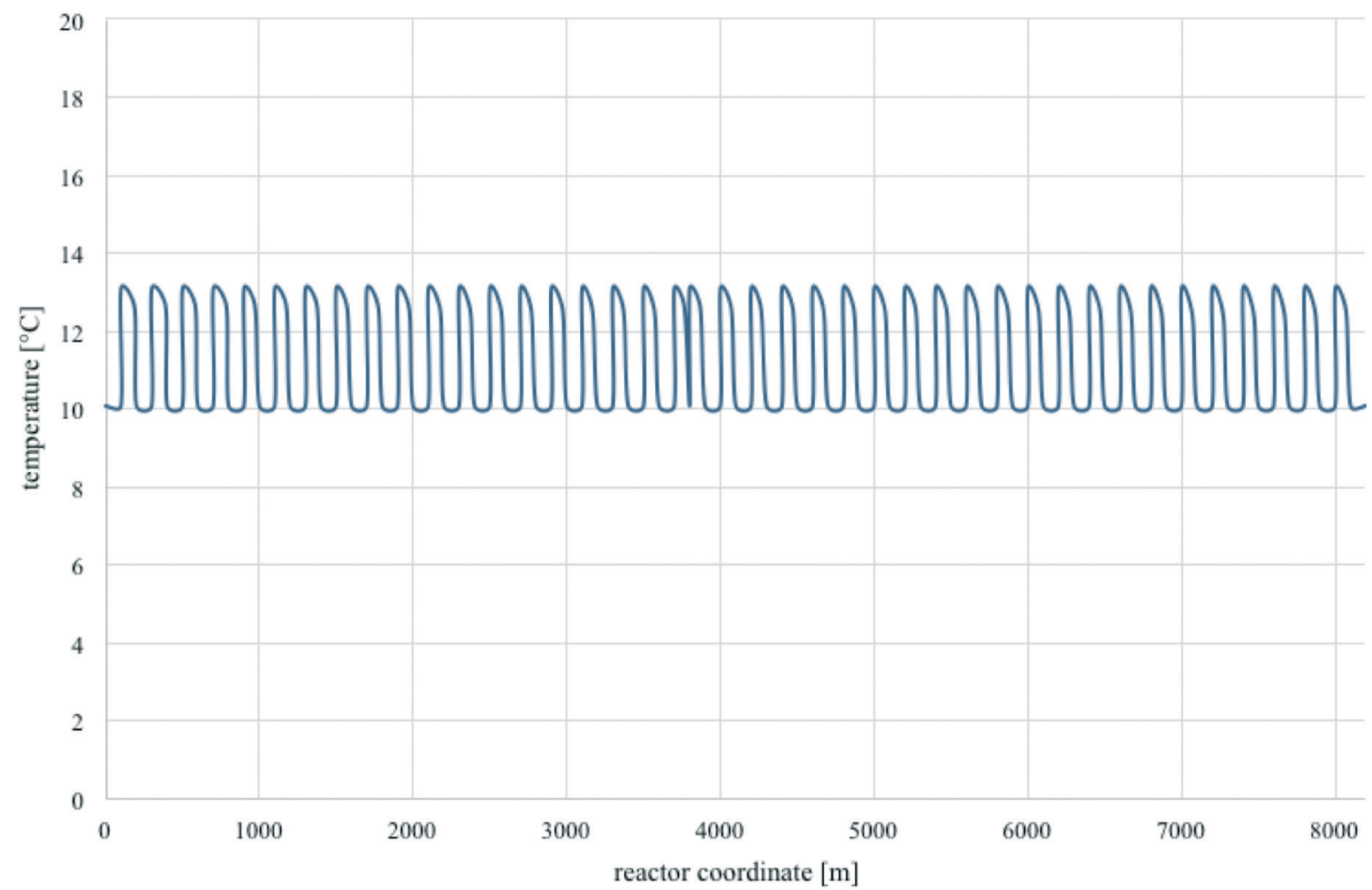

Fig. 5 Trend in the temperature of the slurry along the axial coordinate of the reactor. 82 -Segment reactor of $100 \mathrm{~m}, d_{\mathrm{b}}=1500 \mu \mathrm{m}, P=150$ atm.

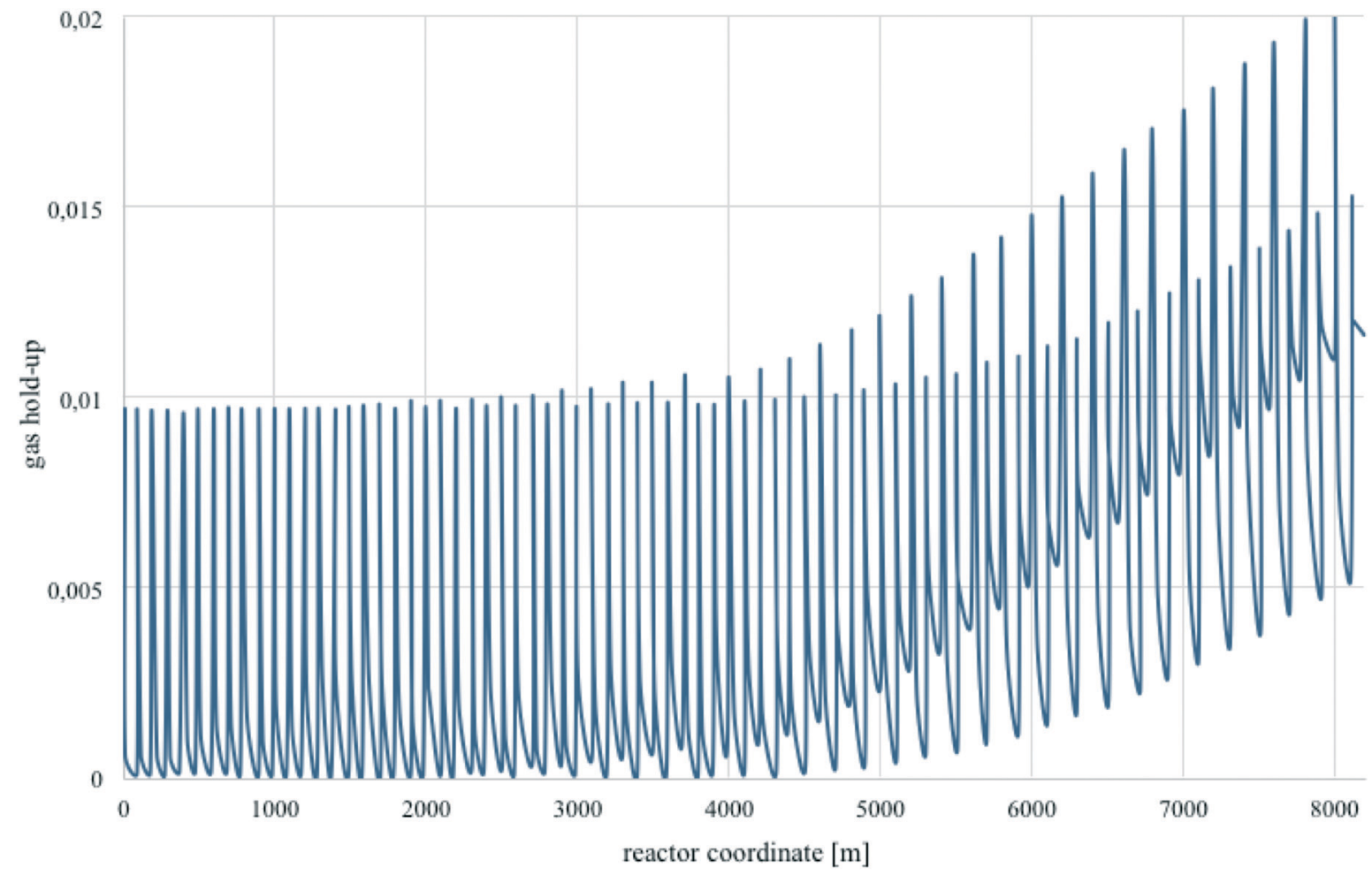

Fig. 6 Trend in the hold-up along the axial coordinate of the reactor. 82-Segment reactor of $100 \mathrm{~m}, d_{\mathrm{b}}=1500 \mu \mathrm{m}, P=150 \mathrm{~atm}$. 


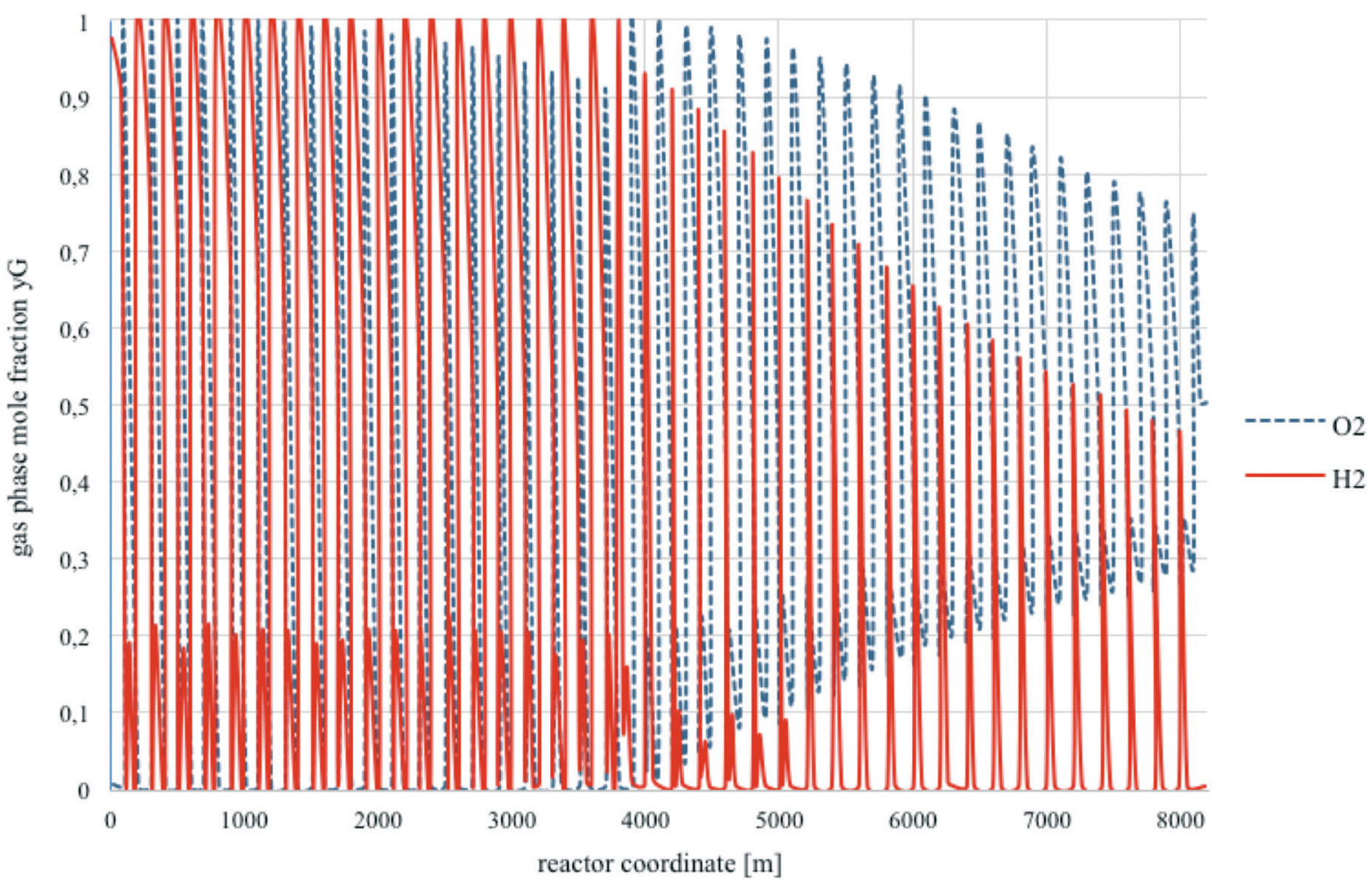

Fig. 7 Trend in the composition of the gaseous phase on the axial coordinate of the reactor. 82 -Segment reactor of $100 \mathrm{~m}, d_{\mathrm{b}}=1500 \mu \mathrm{m}, P=$ $150 \mathrm{~atm}$.

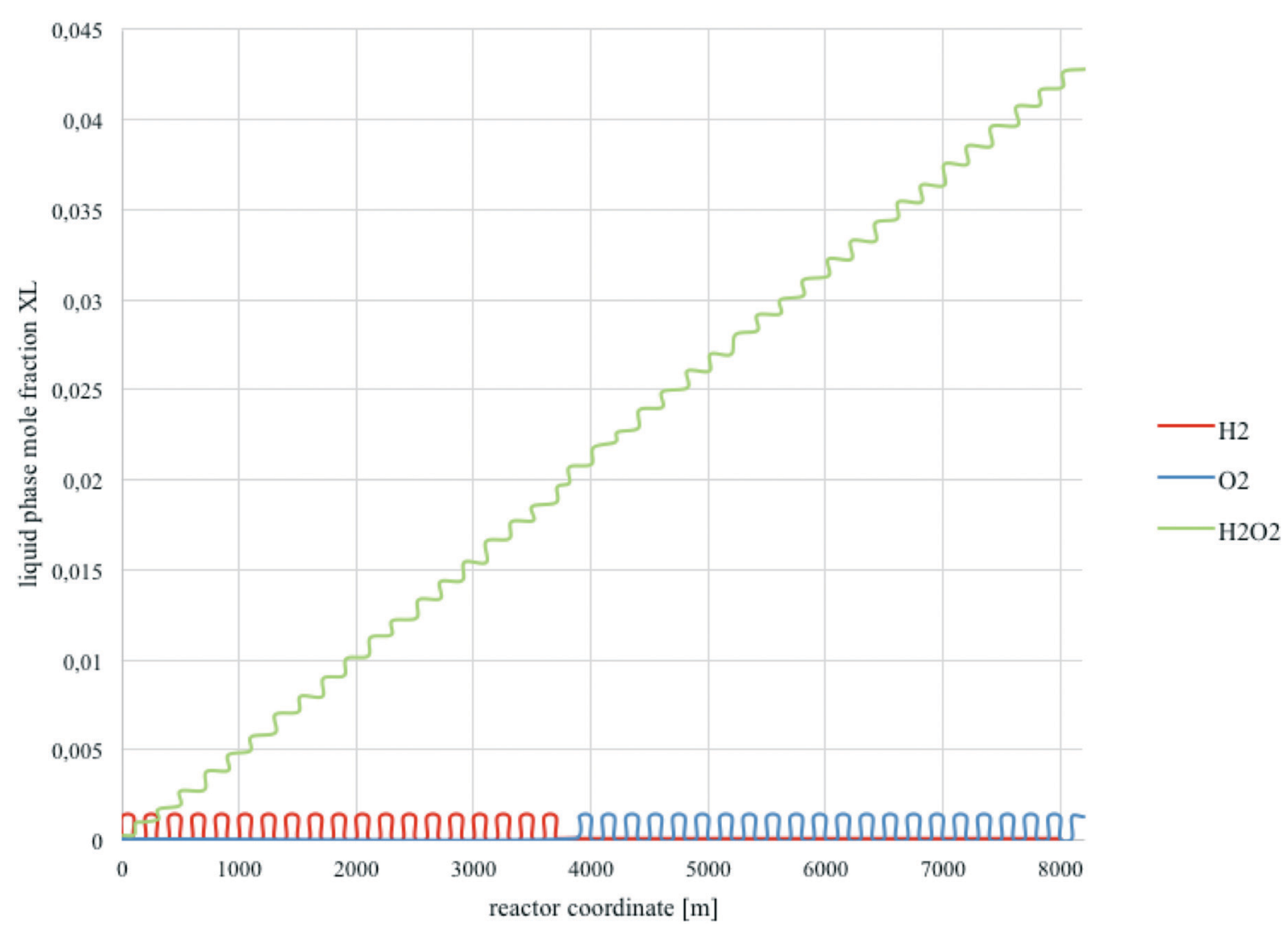

Fig. 8 Trend in the composition of the liquid phase on the axial coordinate of the reactor. 82 -Segment reactor of $100 \mathrm{~m}, d_{\mathrm{b}}=1500 \mu \mathrm{m}, P=150$ atm. 
previous simulations. Gas hold-up was the only outlier due to an accumulation of inert gases.

Fig. 5 represent the temperature profile. It can be seen how the temperature is completely independent from the number of stages, always having the same cyclic pattern. In Fig. 6, gaseous hold-ups can be observed. Here the trend is slightly different from what can be deduced from the previous figure: this is due to an accumulation of inert gases inside the reactor, introduced as impurities together with the reactant species. In Fig. 7 and 8, the molar fractions in the gaseous and liquid phase, respectively, are plotted. As already discussed for Fig. 5, also here it is possible to observe a cyclic pattern. These oscillations allow us to say that the overall behavior of the process did not change when varying the number of reactor segments used. After a few sections, the reactor entered a condition of stationary working, and the quantity of hydrogen peroxide produced was directly proportional to the quantity of gas fed to the reactor. It should be of interest analyzing the first 400 meters and to observe what effect various partitioning would have on the results. The configurations analyzed were the following: four segments of $100 \mathrm{~m}$ each, eight segments of $50 \mathrm{~m}$ each, and twenty segments of $20 \mathrm{~m}$ each. For each case, a slurry flow rate of $65 \mathrm{~m}^{3} \mathrm{~h}^{-1}$ (5\% weight of catalyst), a gas inlet flow rate of $100 \mathrm{Nm}^{3} \mathrm{~h}^{-1}$, and an initial pressure of 150 atm were considered. The productivity of the reactor will be different for each scenario, since the moles of fueling gaseous reagent are different. We just focused on identifying the periodic working conditions and controlled how the reaction contributed to the consumption of the gases, i.e., by decreasing the required length of consumption. It was of pivotal importance that all the operations were done under limited gaseous hold-up conditions. It was noted that an increase in the number of reactor segments increased the productivity of hydrogen peroxide; this was due to enhancing the number of injection points, thus incrementing the number of reacting moles. Given that the process was controlled by the rate of mass transfer, when both reagents encountered each other on the catalyst, they immediately reacted. Even if for segments with a length of $100 \mathrm{~m}$ the gaseous phase is completely dissolved before the following injection, it is different with shorter tubes.

If the $20 \mathrm{~m}$ long segment is chosen, the gas hold-up will have an oscillating behavior between $1.5 \%$ and $2.5 \%$, with a bubble composition of hydrogen in oxygen of between $40 \%$ and $60 \%$; thus the reactor always works within the flammability limits. Moreover $400 \mathrm{~m}$ of reactor produced a liquid phase containing $1.7 \%$ hydrogen peroxide: to obtain a solution at $7 \%, 1680 \mathrm{~m}$ was necessary, while for one at $15 \%, 3560 \mathrm{~m}$ was needed. In order to have a kilometric reactor in reality, the different segments cannot be simply put together in series. Here, a solution could be toggling between vertical ascending and descending segments, thus reducing the space and allowing a more compact layout.

\section{Conclusions}

The simulations carried out showed the feasibility of a process based on the direct synthesis of hydrogen peroxide. If the reactor size is properly designed, it is possible to obtain a certain productivity, in particular:

- The length of each segment depends basically on the interphase mass-transfer efficiency, considering that the stage that kinetically controls the process is the gas/liquid mass transfer.

- The diameter of the reactor depends on the flow dynamics and stability of the bubble motion (i.e., superficial velocities $>2 \mathrm{~m} \mathrm{~s}^{-1}$ ).

- The number of segments depends on the amount of gas that can be adsorbed by the catalyst, a function basically of the amount of catalyst dispersed and its loading capacity.

The data used in the simulations herein were all within reliable limits, as they are known from literature or were estimated with proper equations. Most uncertainties arise from determining the kinematic and thermodynamic data of adsorption, for which it is necessary to experimentally forecast them with the help of a reference catalyst. It is also possible to operate the reactor inside the flammability limits by reducing its size. However, it is unhelpful to reduce the number of injection points because this will also reduce the catalyst usage efficiency. Furthermore this should be matched with an increase in the inlet gas flow rate, thus increasing the hold-ups.

\section{Conflicts of interest}

There are no conflicts to declare.

\section{References}

1 Y. Voloshin and A. Lawal, Chem. Eng. Sci., 2010, 65, 1028-1036.

2 E. Santacesaria, M. Di Serio, R. Velotti and U. Leone, Ind. Eng. Chem. Res., 1994, 33, 277-284.

3 T. Kilpiö, P. Biasi, A. Bittante, T. Salmi and J. Wärnå, Ind. Eng. Chem. Res., 2012, 51, 13366-13378.

4 V. Paunovic, J. C. Schouten and T. A. Nijhuis, Appl. Catal., A, 2015, 505, 249-259.

5 I. Yamanaka, R. Ichihashi, T. Iwasaki, N. Nishimura, T. Murayama, W. Ueda and S. Takenaka, Electrochim. Acta, 2013, 108, 321-329.

6 I. Yamanaka, T. Onizawa, H. Suzuki, N. Hanaizumi, N. Nishimura and S. Takenaka, J. Phys. Chem. C, 2012, 116, 4572-4583.

7 M. Giomo, A. Buso, P. Fier, G. Sandonà, B. Boye and G. Farnia, Electrochim. Acta, 2008, 54, 808-815.

8 E. Pizzutilo, O. Kasian, C. H. Choi, S. Cherevko, G. J. Hutchings, K. J. J. Mayrhofer and S. J. Freakley, Chem. Phys. Lett., 2017, 683, 436-442. 
9 S. Quon, D. Y. Jo, G. H. Han, S. S. Han, M. gi Seo and K. Y. Lee, J. Catal., 2018, 368, 237-247.

10 S. J. Freakley, M. Piccinini, J. K. Edwards, E. N. Ntainjua, J. A. Moulijn and G. J. Hutchings, ACS Catal., 2013, 3, 487-501.

11 Y. Voloshin, R. Halder and A. Lawal, Catal. Today, 2007, 125, 40-47.

12 M. Selinsek, A. Pashkova and R. Dittmeyer, Catal. Today, 2015, 248, 101-107.

13 N. Gemo, P. Biasi, P. Canu and T. O. Salmi, Chem. Eng. J., 2012, 207-208, 539-551.

14 T. Deguchi, H. Yamano and M. Iwamoto, Catal. Today, 2015, 248, 80-90.

15 I. Huerta, J. García-Serna and M. J. Cocero, J. Supercrit. Fluids, 2013, 74, 80-88.

16 T. Moreno, J. García-Serna and M. J. Cocero, J. Supercrit. Fluids, 2011, 57, 227-235.

17 P. Biasi, N. Gemo, J. R. Hernández Carucci, K. Eränen, P. Canu and T. O. Salmi, Ind. Eng. Chem. Res., 2012, 51, 8903-8912.

18 P. Tian, X. Xu, C. Ao, D. Ding, W. Li, R. Si, W. Tu, J. Xu and Y. F. Han, ChemSusChem, 2017, 10, 3342-3346.

19 N. Gemo, T. Salmi and P. Biasi, React. Chem. Eng., 2016, 1, $300-312$.
20 M. Selinsek, M. Bohrer, B. K. Vankayala, K. Haas-Santo, M. Kraut and R. Dittmeyer, Catal. Today, 2016, 268, 85-94.

21 I. John, S. Batchelder and S. S. Crump, US Pat., US005641467A, 1999.

22 R. B. Bird, W. E. Stewart and E. N. Lightfoot, Transport Phenomena, 2nd edn, 2004.

23 Ullmann's Encyclopedia of Industrial Chemistry, 1992, vol. B4.

24 N. Kantarci, F. Borak and K. O. Ulgen, Process Biochem., 2005, 40, 2263-2283.

25 R. Perry, S. Perry, D. Green and J. Maloney, Perry's Chemical Engineers' Handbook, 7th edn, 1997.

26 R. Lemoine and B. I. Morsi, Int. J. Chem. React. Eng., 2005, 3, A20.

27 X. Luo, D. J. Lee, R. Lau, G. Yang and L. S. Fan, AIChE J., 1999, 45, 665-680.

28 M. Polli, M. Di Stanislao, R. Bagatin, E. A. Bakr and M. Masi, Chem. Eng. Sci., 2002, 57, 197-205.

29 V. Semião, P. Andrade and M. D. G. Carvalho, Fuel, 1996, 75, 1707-1714.

30 A. A. C. M. Beenackers and W. P. M. Van Swaaij, Chem. Eng. Sci., 1993, 48, 3109-3139.

31 R. J. Goldston, J. Nucl. Mater., 2015, 463, 397-400.

32 J. L. Waters, J. Chromatogr. Sci., 1971, 9, 428-431.

33 P. E. Barker and G. Ganetsos, Sep. Sci. Technol., 1987, 22, 2011-2035. 